\title{
Improved clearing method contributes to deep imaging of plant organs
}

\section{Yuki Sakamoto}

Tokyo University of Science

\section{Anna Ishimoto}

Tokyo University of Science

\section{Yuuki Sakai}

Kobe University

\section{Moeko Sato}

Yokohama City University

Ryuuichi Nishihama

Tokyo University of Science

Konami Abe

Tokyo University of Science

\section{Yoshitake Sano}

Tokyo University of Science

\section{Teiichi Furuichi}

Tokyo University of Science

\section{Hiroyuki Tsuji}

Kihara Institute for Biological Research, Yokohama City University https://orcid.org/0000-0002-6444$559 \mathrm{X}$

\section{Takayuki Kohchi}

Kyoto University https://orcid.org/0000-0002-9712-4872

Sachihiro Matsunaga ( $\square$ sachi@edu.k.u-tokyo.ac.jp )

University of Tokyo https://orcid.org/0000-0003-3024-3559

\section{Article}

Keywords: Transparent organ, Tissue clearing technology, Deep imaging

Posted Date: June 9th, 2021

DOI: https://doi.org/10.21203/rs.3.rs-563031/v1

License: (a) This work is licensed under a Creative Commons Attribution 4.0 International License. Read Full License 
Version of Record: A version of this preprint was published at Communications Biology on January 10th, 2022. See the published version at https://doi.org/10.1038/s42003-021-02955-9. 


\section{Abstract}

Tissue clearing methods are increasingly essential for microscopic observation of internal tissues of thick biological organs. We previously developed TOMEl, a clearing method for plant tissues; however, it could not entirely remove chlorophylls and reduced the fluorescent signal of fluorescent proteins (FPs). Here, we developed an improved TOMEI method (iTOMEI) to overcome these limitations. We show that iTOMEI efficiently removes chlorophylls using caprylyl sulfobetaine solution and restores fluorescence of FPs, mainly lost by fixation, using a weak alkaline solution. iTOMEl enables detection of much brighter FP fluorescence than previous methods within $26 \mathrm{~h}$ in tissues of Arabidopsis thaliana, Oryza sativa, and Marchantia polymorpha. Moreover, a mouse brain was also efficiently cleared by the iTOMEI-Brain method within $48 \mathrm{~h}$ and strong fluorescent signals were detected in the cleared brain.

\section{Introduction}

The development of microscopes, dyes, fluorescent proteins (FPs), and sample preparation methods have enabled observation of bright and high-resolution microscopic images, which is the driving force to unravel the mystery of life. In particular, FPs are indispensable tools to visualize cellular components, including organelles, proteins, nucleic acids, and small molecules, and provide information such as temperature, $\mathrm{pH}$, and voltage $\mathrm{e}^{1-3}$. However, it is often difficult to sufficiently detect the fluorescent signals of FPs emitted from the internal regions of three-dimensionally thick tissues because autofluorescent pigments absorb the light and some cell components, which refract or reflect light, scatter and disturb the signals. Several clearing methods to elute pigments from cells and adjust the refractive index through the specimen to the mounting medium have been developed to observe the tissue deeply embedded in an organ. The clearing methods developed for animal tissues, including Sca/eS, CUBIC, PACT, and SeeDB2, can maintain the fluorescence of FPs in highly transparent tissues ${ }^{4-8}$. Sca/eS uses the hyperhydration effect of urea to make animal tissues transparent, CUBIC and PACT employ effective detergents to remove obstacles, and SeeDB2 uses iohexol solution for transparency. For plant tissues, Sca/e based method $^{9}$, ClearSee $^{10,11}$, PEA-CLARITY ${ }^{12}$, and TOMEI ${ }^{13-15}$ were developed as optical clearing methods and preserve the fluorescence of FPs. In fact, TOMEl is a powerful technique to analyze the network of vascular tissues in plant organs ${ }^{16,17}$. All of these methods substantially ameliorate the optical properties of specimens; however, each method has drawbacks. Scale-based methods, ClearSee, and PEA-CLARITY are slow to complete clearing. Although TOMEI rapidly clears plant tissues and organs ${ }^{14,15}$, certain autofluorescent pigments remain in plant tissues after clearing and a high concentration of 2,2'thiodiethanol (TDE), used in the TOMEl procedure, reduces the fluorescence intensity of FPs ${ }^{13,18}$.

To overcome the problem of autofluorescence from remnant pigments, we modified all steps of the TOMEI method, including fixation, decolorization, FP reactivation, and mounting, and propose here an improved version of TOMEl, which we designate iTOMEI. We successfully performed deep imaging in plant tissues of Arabidopsis thaliana, Oryza sativa, and Marchantia polymorpha as well as mouse brain tissue using iTOMEl. 


\section{Results}

\section{Assessment of fixation conditions to maintain fluorescence of FPs}

To improve TOMEI, we first reconsidered the fixation conditions to prevent the reduction in fluorescence of FPs. We investigated the effect of five buffers (PBS, PIPES, HEPES, MOPS, and Tris-HCl) adjusted to $\mathrm{pH} 7.0$ using GFP fluorescence, which is unstable under an acidic $\mathrm{pH}$. Arabidopsis thaliana seedlings overexpressing GFP were fixed with $2 \%$ formaldehyde (FA) in these buffers. The GFP fluorescence in the hypocotyl was detected using a fluorescence stereomicroscope before and after fixation. PBS and PIPES maintained GFP fluorescence comparatively better than HEPES, MOPS, and Tris- $\mathrm{HCl}$ (Fig. 1a). A 4\% FA solution was used for plant tissue fixation; however, it dramatically reduced GFP fluorescence even in PBS (Fig. 1b). Fixation with 1\% FA more effectively maintained fluorescence, although the fluorescence intensity was reduced to $40 \%$ compared with that before fixation (Fig. $1 \mathrm{~b}$ ). To examine the effect of $1 \% \mathrm{FA}$ fixation on protein localization and cellular structures, the subnuclear localization of a proliferating cell nuclear antigen (PCNA) fused with EGFP was observed after fixation. The PCNA functions as a sliding clamp to tether DNA polymerase to DNA and PCNA-GFP shows nuclear speckles during the late S-phase in living $A$. thaliana cells (Fig. 1C) ${ }^{19,20}$. Nuclear speckles were not detected after treatment with $0.1 \% \mathrm{FA}$ because such a low concentration of FA was insufficient for fixation. However, treatment with $1 \%, 2 \%$, and 4\% FA maintained the subnuclear and speckle localization of PCNA, suggesting that $1 \%$ FA is sufficient to fix cellular structures and the best fixation condition to preserve GFP fluorescence in our clearing method (Fig. 1c).

\section{Caprylyl sulfobetaine elutes chlorophylls without GFP quenching}

The autofluorescence of chlorophylls derived from chloroplasts is an obstacle for deep imaging of plant tissues. To identify a reagent for efficient removal of chlorophylls, we screened 19 detergents. Fixed 2week-old seedlings of $A$. thaliana were incubated in PBS buffer supplemented with $10 \%$ different detergents for $24 \mathrm{~h}$. The absorbance of chlorophylls, which were eluted from the seedling to the detergent solution, was measured by an absorption spectrophotometer. We determined caprylyl sulfobetaine (\#7) and sodium deoxycholate (\#3) to be the best and second-best detergents for efficiency in chlorophyll elution (Fig. 1d). To investigate their effect on GFP fluorescence, fixed wild-type plants and plants overexpressing GFP were incubated with 10\% caprylyl sulfobetaine and sodium deoxycholate for $24 \mathrm{~h}$. Both detergents slightly enhanced GFP fluorescence after incubation (Fig. 1e). This tendency of sodium deoxycholate is consistent with a previous report for ClearSee ${ }^{10}$. Our assessment suggested that caprylyl sulfobetaine is the best detergent for chlorophyll elution without reducing GFP fluorescence.

To enhance the effect of a zwitterionic detergent, caprylyl sulfobetaine, we tested the combinations of caprylyl sulfobetaine with a nonionic detergent, Triton X-100 (Fig. 1f), an anionic detergent, sodium deoxycholate (Fig. 1g), and urea (Fig. 1h). However, all combinations failed to enhance chlorophylls 
elution, suggesting that incubation with caprylyl sulfobetaine alone is the best. Subsequently, chlorophyll elution efficiency and GFP fluorescence were measured after incubation with PBS supplemented with $10 \%, 20 \%$, or $30 \%$ caprylyl sulfobetaine. The PBS buffer with $20 \%$ caprylyl sulfobetaine showed the highest efficiency of chlorophyll elution and maintenance of GFP fluorescence, indicating that $20 \%$ caprylyl sulfobetaine was optimal for our clearing technology.

\section{Alkaline buffers chemically reactivate FP fluorescence in plant tissues}

Chemical reactivation (CR) enables recovery of reduced GFP fluorescence in resin-embedded specimens by alkaline treatment in animal tissues. We tested the effect of CR on GFP fluorescence in A. thaliana. Fixed seedlings were treated with trisodium phosphate buffer at $\mathrm{pH} 10.7$ and sodium carbonate buffer at $\mathrm{pH} 11.3$ for $2 \mathrm{~h}$. Both treatments significantly enhanced GFP fluorescence in fixed plants overexpressing GFP after CR (Fig. 2a), demonstrating that CR recovered the reduced fluorescence of GFP in plants. In addition, reactivated GFP fluorescence after CR was maintained in PBS buffer for $3 \mathrm{~h}$ (Fig. 2a). To determine the optimum buffer $\mathrm{pH}$ for $\mathrm{CR}$, we modified the $\mathrm{pH}$ of sodium dihydrogen phosphate buffer, sodium hydrogen phosphate buffer, and trisodium phosphate buffers to prepare buffers ranging in $\mathrm{pH}$ from 8.0 to 12.0. Fixed seedlings were incubated in the buffers at $\mathrm{pH} 7.4$ to 12.0 for $2 \mathrm{~h}$. Buffers at $\mathrm{pH} 8.0$ to 11.0 recovered the GFP fluorescence to the same degree (Fig. 2b), thus we chose the pH 8 buffer for our clearing method.

Next, aiming to simplify the process and reduce the procedure time, we attempted to combine decolorization with $\mathrm{CR}$. Fixed seedlings were incubated in PBS buffer at $\mathrm{pH} 7.4$ or sodium phosphate buffer at pH 8.0 supplemented with $10 \%$ caprylyl sulfobetaine for $24 \mathrm{~h}$. In addition, the seedlings were incubated in PBS buffer at $\mathrm{pH} 7.4$ with $10 \%$ caprylyl sulfobetaine for $24 \mathrm{~h}$ and sequentially incubated in sodium phosphate buffer at pH 8.0 for $2 \mathrm{~h}$ (Fig. 2c). Incubation at pH 8.0 with $10 \%$ caprylyl sulfobetaine for $24 \mathrm{~h}$ resulted in GFP fluorescence of higher intensity than other incubations (Fig. 2c). Thus, decolorization and $\mathrm{CR}$ were performed simultaneously with this method.

\section{lohexol enables clearing of plant organs without FP quenching}

Finally, we searched for a clearing reagent superior to TDE, which is used for TOMEl. lohexol dramatically improves the transparency of mouse brain tissue without FP quenching ${ }^{7}$. We investigated whether iohexol improves clarity and does not affect GFP fluorescence in plant tissues. Fixed and decolorized seedlings were incubated in PBS buffer, 97\% (w/w) TDE, and 70.4\% (w/w) iohexol solution for $1 \mathrm{~h}$, and then GFP fluorescence intensity was measured. TDE slightly decreased GFP fluorescence compared with PBS buffer, whereas iohexol treatment did not affect GFP fluorescence (Fig. 2d). Subsequently, the transparency of the cleared leaf was compared by measuring the intensity of grids viewed through the leaf (Fig. 2e). TDE and iohexol decreased the intensity of grids behind the leaf compared with that of PBS buffer (Fig. 2e). These results suggested that iohexol is also a suitable clearing reagent for plant tissues. 
The final protocol of iTOMEl is as follows. Step 1: a sample is fixed in PBS buffer supplemented with 1\% FA for $1 \mathrm{~h}$. Step 2: the fixed sample is decolorized in sodium phosphate buffer at pH 8.0 supplemented with $20 \%$ caprylyl sulfobetaine for $24 \mathrm{~h}$. Step 3: the sample is incubated in $70.4 \%$ iohexol solution for $1 \mathrm{~h}$.

\section{ITOMEl is the best clearing technique for plant organs within $26 \mathrm{~h}$}

The iTOMEI procedure was compared with the clearing methods ClearSee and TOMEI-II. The total processing time for each technique was unified to $26 \mathrm{~h}$. iTOMEl-treated seedlings of $A$. thaliana showed the highest transparency (Fig. 3a). Seedlings overexpressing GFP were fixed in PBS buffer supplemented with $1 \%, 2 \%$, or $4 \%$ FA and treated with the three clearing methods. iTOMEI maintained the highest fluorescence intensity among the three methods under each fixation condition (Fig. 3b). Confocal fluorescence images of cotyledons in seedlings expressing histone H2B-GFP were captured after applying the three clearing techniques (Fig. 3c). The fluorescent signals were almost invisible at depths of 45 and $75 \mathrm{~mm}$ in cotyledons treated with PBS buffer and TOMEI-II, respectively. Although GFP signals were detected at $75 \mathrm{~mm}$ depth in cotyledons cleared using ClearSee or iTOMEI, the brightest fluorescence at 75 $\mathrm{mm}$ depth in the cotyledon was detected after iTOMEI treatment.

\section{iTOMEI reveals the FP expression pattern inside plant tissues and organs}

Next, we performed iTOMEI to clear organs of rice (Oryza sativa). Three days was required for the leaves to become completely transparent (Fig. 4a). We also observed the expression pattern of the transcription factor OsMADS15, which regulates the transition from the vegetative to the reproductive phase in the shoot apical meristem (SAM). Given that the diameter of the rice reproductive SAM is approximately 150 $\mu \mathrm{m}$, the FP expression pattern in the rice SAM could not be analyzed without sectioning. After $4 \%$ FA fixation of the SAM dissected from plants expressing OsMADS15 fused with mOrange ${ }^{22}$, the fixed SAM was stained with a fluorescent cell-wall-staining optical brightener, SCRI Renaissance 2200 (SR2200), and cleared with TOMEI-II or ITOMEI. Neither fluorescent signal was detected in the central area of the SAM in only fixed SAMs (Fig. 4b). The SR2200 signal was detected in the central portion of the SAM in TOMEI-IItreated samples, whereas OsMADS15-mOrange signal was not detected at the depth of $80 \mu \mathrm{m}$. After clearing with iTOMEl, both signals were detected in the central region of the SAM at $80 \mu \mathrm{m}$ depth.

OsMADS15-mOrange was strongly expressed in the basal region of a hairy bract and in the outer two cell layers of an incipient primary branch meristem (Fig. 4b), suggesting that iTOMEI can reveal the expression pattern of FPs inside the larger reproductive SAM. We also attempted to detect auxin signaling in the root tips of plants expressing pDR5rev::NLS-3xVenus. iTOMEI visualized pDR5rev::NLS-3xVenus in the root tip, whereas PBS alone did not (Fig. 4c). The pDR5rev::NLS-3xVenus construct was strongly expressed in the central and surrounding metaxylem cells in the stele, quiescent center, and columella cells. In an optical cross-section of the root, five FP foci surrounding the signal in the center of the root were detected, corresponding to the central and surrounding metaxylem (Fig. 4d). 
Next, we attempted to clear organs of the liverwort Marchantia polymorpha. An apical portion of a mature thallus of M. polymorpha was cleared within $26 \mathrm{~h}$ as for $A$. thaliana seedlings (Fig. 5a). Thalli of a 2week-old gemmaling expressing histone H2B-tdTomato were fixed and treated with PBS, TOMEI-II, and iTOMEI. tdTomato signals were observed from the dorsal to the ventral surface in the thalli by confocal microscopy. Fluorescent signals were detected at the depth of $200 \mu \mathrm{m}$ in the iTOMEl-treated gemmaling, but not in PBS- and TOMEI-II-treated gemmalings (Fig. 5b). Calcofluor White M2R and H2B-tdTomato signals were observed from the ventral to the dorsal surface of the thallus in a 3-day-old gemmaling (Supplementary Fig. 1). In the apical region of the thallus, we detected an apical cell or subapical cells that appeared fan-shaped when viewed in the $Y-Z$ optical section (Fig. $5 \mathrm{c})^{23}$. These observations demonstrated that iTOMEl is suitable for deep imaging of M. polymorpha thalli.

$R S L$ class I genes encode basic helix-loop-helix transcription factors conserved among land plants. These transcription factors positively regulate root hair formation in $O$. sativa and $A$. thaliana, and rhizoid development in M. polymorpha and Physcomitrium patens ${ }^{24-27}$. RSL class I genes are expressed in a precursor cell of the root hair and rhizoid in $O$. sativa, A. thaliana, and P. patens. However, the expression pattern of an ortholog of $M$. polymorpha, MpRSL 1, has not been investigated. The expression pattern of nuclear tdTomato under control of the MpRSL 1 promoter in the gemma showed that the promoter was activated in rhizoid precursor cells as expected and, unexpectedly, in the apical regions (Fig. 5d). Thus, MpRSL1 may function in the apical region of gemmae. Next, the gemma cup, a cup-shaped receptacle on the mature thallus, was cleared using iTOMEl. Under observation with a two-photon excitation microscope, the gemmae were observed within the transparent gemma cup through the wall of the gemma cup (Fig. 5e). Many immature gemmae are present in a gemma cup and do not develop synchronously, which enables observation of gemmae at various developmental stages within a gemma cup (Fig. 5f). MpRSL 1 was strongly expressed in a preapical region of the immature gemmae at different developmental stages (Fig. 5f, arrowheads), and MpRSL 1 expression was detected in rhizoid precursor cells in large immature gemmae (Fig. $5 f$, arrows) but not in smaller gemmae. These results demonstrate that iTOMEl enables in situ observation of gene expression patterns during gemma development through the wall tissues of the gemma cup.

\section{ITOMEI contributes to deep imaging of mouse brain}

Finally, we tested whether the range of application of iTOMEI could be expanded to animal tissues. We modified the iTOMEI protocol for the mouse brain with reference to the SeeDB2 protocol and designated the procedure iTOMEI-Brain (iTOMEI-B). Fixed mouse brain was sliced at $2 \mathrm{~mm}$ and treated with PBS, SeeDB2, and iTOMEI-B. Although 24-hour treatment of iTOMEI-B was insufficient to clear the brain, 48hour treatment achieved high transparency (Supplementary Fig. 2a). iTOMEI-B inhibited the size change of the slice as much as SeeDB2 (Supplementary Fig. 2a). Mouse brain expressing EGFP was also treated with PBS, SeeDB2, and iTOMEI-B, and observed from the cortical surface of the brain using a two-photon excitation microscope. iTOMEI-B allowed acquisition of fluorescence images to $3 \mathrm{~mm}$ depth in transparent brain expressing GFP (Supplementary Fig. 2b). The GFP fluorescence was clearly detected in the soma, axon, and dendrite of the hippocampus and cerebral cortex (Supplementary Fig. 2b-d). 
Surprisingly, the axonal projection was observed in the thalamus at the depth of $3 \mathrm{~mm}$ from the cortical surface. Deep imaging of the transparent brain using iTOMEI-B enabled identification of the layer structure of the hippocampus and cortex. The characteristic dendritic morphology of CA1 pyramidal neurons was maintained (Supplementary Fig. 2d). Our imaging data suggested that iTOMEI can be adapted to not only plant tissues but also the mouse brain.

\section{Discussion}

The iTOMEI procedure can preserve and recover bright FP fluorescence of improved intensity in transparent organs and tissues compared with previous methods. This advantage enables threedimensional detection of weak fluorescent signals from FP in internal tissues of thick specimens. Chemical reactivation enhances the fluorescence intensity of GFP and YFP in resin-embedded biological tissues ${ }^{21}$. Alkaline solutions in the range from $\mathrm{pH} 9.0$ to 12.0 are generally used for CR. Given that biological tissues are morphologically damaged by a solution at such a high $\mathrm{pH}$, adaptation of $\mathrm{CR}$ for sample preparation without embedding in resin seemed difficult. However, we showed that a weak alkaline solution of $\mathrm{pH} 8.0$ harbors the fluorescence recovery capability (Fig. 2b). In addition, PBS buffer at $\mathrm{pH} 7.4$ slightly reactivated fluorescence. These results suggested that sample incubation in solutions at $\mathrm{pH} 7.0$ to 8.0 can reactivate FPs quenched by fixation.

Fixation greatly reduces the fluorescence intensity of GFP in $A$. thaliana. The fluorescence intensity of a sample fixed with $1 \%$ FA was approximately double and four-times higher than that of samples fixed with $2 \%$ and $4 \%$ FA, respectively (Fig. 1b). The final fluorescence intensity was less affected by FA concentration because the fluorescent intensity of iTOMEI-treated samples fixed with $1 \%$ FA was not more than double that of iTOMEI-treated samples fixed with 4\% FA (Fig. 3b). ClearSee and TOMEI-II showed a similar tendency to iTOMEl, suggesting that the GFP inactivated by high FA concentration was easily recovered during each procedure because incubation in near-neutral solutions can recover GFP fluorescence.

Clearing of the rice SAM by iTOMEI provided spatial expression-profiling of the key transcription factor OsMADS15 at three-dimensional single-cell resolution (Fig. 4). OsMADS15, which is a homolog of APETALA1/FRUITFUL in A. thaliana, is essential for bract and floral organ development in rice ${ }^{28}$. Consistent with the reported function, OsMADS15-mOrange signal was observed at the incipient primary branch meristem, which provides flowers at an advanced stage, and in the basal region of a hairy bract, which is a suppressed leaf produced by the reproductive $\mathrm{SAM}^{29}$. OsMADS15 expression is regulated by the florigen $\mathrm{Hd} 3 \mathrm{a}$ in the rice $\mathrm{SAM}^{22,30}$, thus our results suggested the site of action of rice florigen at single-cell resolution. In the Mprs/1-1 mutant of M. polymorpha, rhizoids do not develop from the ventral surface of the thallus, gemmae and mucilage papillae did not develop in gemma cups, and slime papillae did not develop near the apical region ${ }^{26}$. However, it has not been known whether the Mprs/1-1 mutant shows any defects in the apical region. We revealed that MpRSL 1 promoter activity was detected in rhizoid precursor cells and the apical region in mature gemmae, and was restricted in the apical region 
during gemma development in the gemma cup (Fig. $5 \mathrm{~d}-\mathrm{f}$ ). This imaging data suggest that MpRSL 1 is a useful marker to detect meristematic cells in liverworts.

Although iTOMEl was developed for plant tissues and organs, it can be applied to the mouse brain (Supplementary Fig. 2). Because each clearing method has its own characteristics, the method should be used properly depending on what is important in the imaging analyses. For example, the transparency in the brain treated with SeeDB2 was lower compared with iTOMEI-B but the clearing brain by SeeDB2 will highly preserve the cell morphology and the fluorescence of FPs and minimizes the spherical aberration in super-resolution 3D imaging ${ }^{7}$. The high transparency by iTOMEI-B potentially contributes to observation of the weak FP expression in the cells located deep in the brain.

Recent development in transparent techniques enables us to analyze the expression pattern with FPs in the whole tissues and organs. Our developed iTOMEl is a powerful technique to transparentize thick organs with almost no attenuation of the fluorescence from FPs, which were expressed in the profound depth of organs. It will be also a helpful technique to construct the image platform of organ morphology at single-cell resolution.

\section{Material And Methods}

\section{Plant Materials and Growth Conditions}

Arabidopsis thaliana ecotype Columbia-0 was used as the wild type. The transgenic lines $p 355$.:GFP, pPCNA1::PCNA1-EGFP, and p35S::H2B-GFP were previously reported ${ }^{13,20}$. Seeds were germinated and grown on half-strength Murashige and Skoog plates supplemented with $1 \%$ sucrose and 14-day-old plants were transferred to soil. Plants were grown in a growth chamber $\left(16 \mathrm{~h}\right.$ light $/ 8 \mathrm{~h}$ dark, $\left.22^{\circ} \mathrm{C}\right)$.

Oryza sativa 'Nipponbare' was used for SAM analysis. Plants were grown in the growth chamber under short-day conditions $\left(10 \mathrm{~h}\right.$ light at $27^{\circ} \mathrm{C}$ and $14 \mathrm{~h}$ dark at $25^{\circ} \mathrm{C}$, light intensity $400-700 \mathrm{~nm}, 100 \mu \mathrm{mol} \mathrm{m}^{-}$

$\left.2 \cdot s^{-1}\right)$. Vegetative and reproductive SAMs were isolated by hand dissection of the basal region of rice under a microscope ${ }^{31}$. Generation of the transgenic lines OSMADS15-mOragene and DR5rev:NLS-3xVenus was previously reported 22,32 .

A male accession of $M$. polymorpha Takaragaike-1 (Tak-1) was used as the wild type. Plants were grown on half-strength Gamborg's B5 plates in a growth chamber ( $16 \mathrm{~h}$ light $/ 8 \mathrm{~h}$ dark, $\left.22^{\circ} \mathrm{C}\right)$.

\section{Plasmid Construction And Transformation}

The MpRSL 1 (Mp3g17930) genomic region, including a 6964-bp fragment upstream of the 15th Met codon in the first exon, was amplified as the promoter region from Tak-1 genomic DNA by PCR sing a gene-specific primer pair (proMpRSL1-F1, CACCCCCAAATGCAATTCTATTGTGTATTCAT; proMpRSL1-R, AGCTGGGTCGGCGCGCATGTTGTTCCTCCTGCTCAGTGT) and subcloned into the pENTR/D-TOPO vector 
(Thermo Fisher Scientific, USA). A DNA cassette including the promoter region was transferred to a binary vector pMpGWB116 $6^{33}$ using the Gateway LR Clonase II Enzyme mix (Thermo Fisher Scientific). To create a construct for proMpEF1:H2B-tdTomato, a Sall-Notl fragment including a coding sequence for H2BtdTomato from the plasmid spUC-RPS5A::H2B-tdTomato ${ }^{34}$ was ligated with Sall- and Notl-digested pENTR-1A vector (Thermo Fisher Scientific), and the resulting vector was used for LR reaction with a binary vector pMpGWB303 ${ }^{33}$. The binary vectors were used for Agrobacterium-mediated transformation of Tak-1 thalli and Tak-1 - Tak-2 F1 sporelings, respectively.

\section{Measurements of fluorescence intensity in $A$. thaliana}

The fluorescent images of seedlings were captured under the same optical condition using a fluorescence stereomicroscope (SMZ18; Nikon, Japan) equipped with a DS-Ri2 digital camera (Nikon) before and after treatment. The fluorescence intensity of GFP was measured in a hypocotyl of the seedling using ImageJ software. We modified the $\mathrm{pH}$ of sodium phosphate buffer to prepare buffers at $\mathrm{pH} 8.0$ by mixing sodium hydrogen phosphate solution and sodium dihydrogen phosphate solution, sodium hydrogen phosphate solution at $\mathrm{pH} 9.0$ by $\mathrm{NaOH}$, and trisodium phosphate solution at $\mathrm{pH} 10,11$, and 12 by $\mathrm{NaOH}$.

\section{Measurements of chlorophyll absorbance}

Fourteen-day-old seedlings were fixed with 2\% FA in PBS buffer for $1 \mathrm{~h}$. A fixed seedling was placed into a plastic tube containing various detergent solutions and was incubated for $24 \mathrm{~h}$. As a negative control, the detergent solutions without the seedling were incubated for $24 \mathrm{~h}$. The absorbance of chlorophylls, eluted from the seedling to the detergent solution, was measured using an absorption spectrophotometer (Nanophotometer Pearl; IMPEL, Germany).

\section{Optical clearing of $A$. thaliana by iTOMEI}

Seedlings and leaves were fixed in PBS buffer with $1 \%, 2 \%$, and $4 \%$ FA for $1 \mathrm{~h}$ with evacuation for the first $10 \mathrm{~min}$ and then the samples were washed three times in PBS buffer for $5 \mathrm{~min}$. The fixed samples were treated with a decolorization solution ( $100 \mathrm{mM}$ sodium phosphate buffer [a mixture of sodium hydrogen phosphate and sodium dihydrogen phosphate] at $\mathrm{pH} 8.0$ with $20 \%$ caprylyl sulfobetaine [ $\mathrm{TCl}$, Japan]) for $24 \mathrm{~h}$ with gentle shaking. After washing in PBS buffer for $5 \mathrm{~min}$, the samples were incubated in a mounting solution (70.4\% iohexol [TCl or Merck, USA] in PBS buffer) for $1 \mathrm{~h}$ with gentle shaking. Finally, the samples were mounted on a glass slide in the mounting solution and a coverslip applied. Exposure of the mounting solution to air for several minutes results in water evaporation and a skin forms on the solution surface. To inhibit skin formation, the mounting procedure should be conducted rapidly. As the mounting solution is prone to mold growth, it should be stored at $4^{\circ} \mathrm{C}$. All procedures were performed at $25^{\circ} \mathrm{C}$. 


\section{Optical clearing of $\boldsymbol{A}$. thaliana by TOMEI-II}

Seedlings and leaves were fixed in PBS buffer with $1 \%, 2 \%$, and $4 \%$ FA for $1 \mathrm{~h}$ with evacuation for the first $10 \mathrm{~min}$ and then the samples were washed three times in PBS buffer for $5 \mathrm{~min}$. The fixed samples were gradually treated in PBS buffer with a graded series of Tissue-Clearing Reagent TOMEI (TCI) for 10 min each $(10 \%, 30 \%, 50 \%, 70 \%$, and $100 \%)$. The samples were incubated in $100 \%$ Tissue-Clearing Reagent TOMEl for $25 \mathrm{~h}$ with gentle shaking. Finally, the samples were mounted between the glass slide and the coverslip using $100 \%$ Tissue-Clearing Reagent TOMEl. All procedures were performed at $25^{\circ} \mathrm{C}$.

\section{Optical clearing of $A$. thaliana by ClearSee}

Seedlings and leaves were fixed in PBS buffer with $1 \%, 2 \%$, and $4 \%$ FA for $1 \mathrm{~h}$ with evacuation for the first $10 \mathrm{~min}$ and then the samples were washed three times in PBS buffer for $5 \mathrm{~min}$. The fixed samples were treated in ClearSee (Fujifilm, Japan) for $26 \mathrm{~h}$ with gentle shaking. Finally, the samples were mounted between the glass slide and the coverslip using ClearSee. All procedures were performed at $25^{\circ} \mathrm{C}$.

\section{Optical clearing of 0 . sativa by iTOMEI}

A 5-day-old leaf was fixed with 4\% FA for $1 \mathrm{~h}$ with evacuation for the first $10 \mathrm{~min}$ and the samples were washed three times in PBS buffer for $5 \mathrm{~min}$. The fixed sample was incubated in the decolorization solution for $3 \mathrm{~d}$ with gentle shaking. The solution was exchanged with the mounting solution and the samples were incubated in the mounting solution for $1 \mathrm{~h}$ with gentle shaking.

The SAMs of rice plants were isolated by hand dissection of wild-type and transgenic plants. The isolated SAMs were fixed in PBS buffer with 4\% FA for $1 \mathrm{~h}$ with evacuation for the first $10 \mathrm{~min}$. The samples were stained in $0.1 \%$ (v/v) SR2200 (solution from supplier was considered as 100\%: Renaissance Chemicals, UK) at this step. Then the samples were washed three times in PBS buffer for 5 min for the first washing and $10 \mathrm{~min}$ for the second and third washing. The fixed samples were treated in the decolorization solution for $24 \mathrm{~h}$ with gentle shaking. The solution was exchanged with the mounting solution and the samples were incubated in the mounting solution for $1 \mathrm{~h}$ without shaking. Finally, the samples were mounted between the glass slide and the coverslip using the mounting solution.

\section{Optical clearing of 0 . sativa by TOMEI-II}

The SAMs of rice plants were isolated by hand dissection of wild-type and transgenic plants. The isolated SAMs were fixed in PBS buffer with $4 \%$ FA for $1 \mathrm{~h}$ with evacuation for the first $10 \mathrm{~min}$. The samples were stained in $0.1 \%(\mathrm{v} / \mathrm{v})$ SR2200 (solution from supplier was considered as $100 \%$ : Renaissance Chemicals) at this step. Then the samples were washed three times in PBS buffer for $5 \mathrm{~min}$ for the first washing and 
10 min for the second and third washing. The samples ware incubated in $10 \%, 30 \%, 50 \%$, and $70 \%$ TOMEI-II solution for 10 min sequentially. After clearing, the samples were mounted in $70 \%$ TOMEI-II.

\section{Optical clearing of $M$. polymorpha by iTOMEI}

Thalli, gemmalings, and gemmae were fixed in PBS buffer with $1 \% \mathrm{FA}$ for $1 \mathrm{~h}$ with evacuation for the first $10 \mathrm{~min}$ and then the samples were washed three times in PBS buffer for $5 \mathrm{~min}$. The fixed samples were treated in the decolorization solution for $24 \mathrm{~h}$ with gentle shaking. After washing in PBS buffer for $5 \mathrm{~min}$, the samples were stained in Calcofluor White Stain (Calcofluor White M2R $1 \mathrm{~g} / \mathrm{l}$, Evans blue $0.5 \mathrm{~g} / \mathrm{l}$; Merck) or 3-fold diluted CyStain UV Precise P staining buffer (Sysmex, Japan) containing 4',6-diamidino2-phenylindole (DAPI) for $10 \mathrm{~min}$ and $15 \mathrm{~min}$, respectively. After washing in PBS buffer for $5 \mathrm{~min}$, the samples were incubated in the mounting solution for $1 \mathrm{~h}$ with gentle shaking. Finally, the samples were mounted between the glass slide and the coverslip using the mounting solution. For observation of immature gemmae in gemma cups using a two-photon excitation microscope, the whole gemma cup was mounted and the immature gemmae were observed through the side wall of the gemma cup (Fig. 5e).

\section{Optical clearing of M. polymorpha by TOMEI-II}

Two-week-old gemmalings were fixed in PBS buffer with 4\% FA for $1 \mathrm{~h}$ with evacuation for the first 10 $\mathrm{min}$ and then the samples were washed three times in PBS buffer for $5 \mathrm{~min}$. The fixed samples were gradually treated in PBS buffer with a graded series of Tissue-Clearing Reagent TOMEI (TCI) for 10 min each $(10 \%, 30 \%, 50 \%, 70 \%$, and $100 \%)$. The samples were incubated in $100 \%$ Tissue-Clearing Reagent TOMEl for $25 \mathrm{~h}$ with gentle shaking. Finally, the samples were mounted between the glass slide and the coverslip using $100 \%$ Tissue-Clearing Reagent TOMEl. All procedures were performed at $25^{\circ} \mathrm{C}$.

\section{Mice and Surgery}

All experimental protocols were evaluated and approved by the Regulation for Animal Research at Tokyo University of Science. All experiments were conducted in accordance with the Regulations for Animal Research at the Tokyo University Science. Adult C57BI/6J male (4-5 months old) mice were used. Mice were maintained under a $12 \mathrm{~h}$ light/12 $\mathrm{h}$ dark cycle (light period 07:30-19:30), and ad libitum feeding and drinking conditions. The plasmid AAV8 CaMKIla-EGFP was purchased from UNC Vector Core. We used a titer of approximately $1 \times 10^{12} \mathrm{vg} / \mathrm{ml}$ of EGFP viruses in this study. Mice were mounted in a stereotaxic apparatus, anesthetized with pentobarbital $(80 \mathrm{mg} / \mathrm{kg})$ and subcutaneously injected carprofen $(5 \mathrm{mg} / \mathrm{kg})$ and dexamethasone $(0.2 \mathrm{mg} / \mathrm{kg})$. A 2-mm-diameter craniotomy was performed above the hippocampus. A $0.3 \mu \mathrm{l}$ virus solution was infused using a Hamilton syringe through a glass micropipette at the following coordinates: relative to bregma $(\mathrm{mm})$ : anteroposterior axis (AP): -2.0 , mediolateral axis (ML): 1.4, and dorsoventral axis (DV): 0.6, 1.2, and 1.7 from dura mater, taken from the mouse brain atlas ${ }^{28}$ at a rate of $0.1 \mu \mathrm{l} / \mathrm{min}$. A glass capillary was left in place for an additional $10 \mathrm{~min}$. A 
brain sample was harvested 4 weeks after surgery to allow for recovery and sufficient expression of genes.

\section{Mouse brain sample preparation.}

Mice were deeply anesthetized with pentobarbital and transcardially perfused with $4 \%$ paraformaldehyde in $0.1 \mathrm{M}$ sodium phosphate buffer ( $\mathrm{pH} 7.4)$. The brains were excised, sagittally dissected, and postfixed with the same fixative at $4^{\circ} \mathrm{C}$ overnight. The fixed brains were sectioned at a thickness of $2 \mathrm{~mm}$ with a laser blade to make brain slices.

\section{Optical clearing of mouse brain by iTOMEI-B}

The hemi brain and brain slices were treated with $20 \%$ caprylyl sulfobetaine in $0.1 \mathrm{M}$ sodium phosphate buffer $(\mathrm{pH} 8.0)$ for $16 \mathrm{~h}$. The samples were next treated with $18.7 \%(\mathrm{w} / \mathrm{w})$ iohexol and $20 \%$ caprylyl sulfobetaine in PBS for $6 \mathrm{~h}$. Subsequently, the samples were treated with $28.1 \%(\mathrm{w} / \mathrm{w})$ iohexol and $20 \%$ caprylyl sulfobetaine in PBS for $6 \mathrm{~h}$ and then with $56.2 \%(\mathrm{w} / \mathrm{w})$ iohexol in PBS for $12 \mathrm{~h}$. Finally, the hemi brains were treated with $56.2 \%(\mathrm{w} / \mathrm{w})$ iohexol in PBS for $8 \mathrm{~h}$ and the brain slices were treated with $70 \%$ $(\mathrm{w} / \mathrm{w})$ iohexol in PBS for $8 \mathrm{~h}$. The hemi brains were mounted with Omnipaque 350 and observed by a twophoton excitation microscope. The brain slices were mounted with $70 \%(\mathrm{w} / \mathrm{w})$ iohexol in PBS and observed with a stereomicroscope. All procedures were performed at $25^{\circ} \mathrm{C}$.

\section{Optical clearing of mouse brain by SeeDB2}

The hemi brain and brain slices were treated with $2 \%$ saponin in PBS buffer for $16 \mathrm{~h}$. The samples were next treated with 2\% saponin in a 1:2 mixture of Omnipaque 350 (Daiichi-Sankyo, Japan) and water for 6 h. Subsequently, the samples were treated with $2 \%$ saponin in a 1:1 mixture of Omnipaque 350 and water for $6 \mathrm{~h}$ and then $2 \%$ saponin in Omnipaque 350 for $12 \mathrm{~h}$. Finally, the hemi brains were treated with Omnipaque 350 for $8 \mathrm{~h}$ and the brain slices were treated with $2 \%$ saponin in SeeDB2S for $8 \mathrm{~h}$. The hemi brains were mounted in Omnipaque 350 and observed with a two-photon excitation microscope. The brain slices were mounted with SeeDB2S and observed with a stereomicroscope. All procedures were performed at $25^{\circ} \mathrm{C}$.

\section{Confocal microscopy and two-photon excitation microscopy}

The confocal images of $A$. thaliana and $M$. polymorpha were obtained with a confocal laser-scanning microscope (FV1200; Olympus, Tokyo, Japan) equipped with $405 \mathrm{~nm}, 473 \mathrm{~nm}$, and $559 \mathrm{~nm}$ LD laser lines and a $60 \times 1.40$ N.A. oil immersion objective (PlanApo; Olympus), a 40×1.30 N.A. oil immersion objective 
(UPlanFL; Olympus), and a $20 \times 0.75$ dry objective (UPlanSApo; Olympus). Two-week-old gemmalings of M. polymorpha were observed with a confocal laser-scanning microscope (LSM710; Carl Zeiss, Germany) equipped with a $514 \mathrm{~nm}$ laser line and a $20 \times 0.5$ dry objective (EC Plan-Neofluar; Carl Zeiss). tdTomato fluorescent signals were detected at 552-651 nm.

Transgenic rice plant tissues were visualized using confocal laser-scanning microscopy (TCS SP8; Leica Microsystems, Tokyo, Japan) equipped with $405 \mathrm{~nm}$ and a pulsed white-light laser (WLL) lines and 20x multi-immersion objective lens (PL APO CS2 20×/0.75 IMM CORR HC; Leica Microsystems), a 40× waterimmersion objective lens (PL APO CS2 40×/1.10 W CORR HCX; Leica Microsystems), and a 63× glycerolimmersion objective lens (HC PL APO 63×/1.30 GLYC CORR CS2; Leica Microsystems). For mOrange fluorescence, images were captured at 550-600 nm after excitation at $543 \mathrm{~nm}$ with WLL. For SR2200 fluorescence, images were captured at $410-480 \mathrm{~nm}$ after excitation at $405 \mathrm{~nm}$ with a solid-state laser. After image acquisition, the images were processed using LASX software (Leica Microsystems, Tokyo, Japan).

The fluorescence images of immature gemmae and mouse brains were obtained with an upright twophoton excitation microscope (FVMPE-RS; Olympus) equipped with dual femtosecond pulse lasers (InSight DeepSee Dual-OL; Spectra-Physics, CA, USA) and a 25× 1.00 N.A. objective (XLSLPLN25XGMP; Olympus) and a $10 \times 0.6$ N.A. objective (XLPLN10XSVMP; Olympus). The excitation wavelength for DAPI, GFP, and tdTomato was $700 \mathrm{~nm}, 860 \mathrm{~nm}$, and $1040 \mathrm{~nm}$, respectively. Image processing was performed using Imaris and ImageJ software.

\section{Declarations}

\section{Author contributions:}

Y. Sakamoto and S.M. designed the research; Y. Sakamoto, A.I., Y. Sakai, M.S., R.N., K.A., and Y. Sano performed the research; Y. Sakamoto and A.I. analyzed the data; Y. Sakamoto, Y. Sano, T.F., H.T., T.K., and S.M. wrote the paper.

\section{Acknowledgments}

We thank Dr Kimitsune Ishizaki for providing the H2B-tdTomato entry vector and Dr Yuichiro Watanabe, Dr Takahiro Hamada, Mr Kazutaka Futagami, and Ms Sakiko Ishida for technical support with transformation of $M$. polymorpha. This research was supported by MXT/JSPS KAKENHI 18K14743 and $21 \mathrm{~K} 06247$ to Y. Sakamoto, $20 \mathrm{H} 04884$ to R.N., and $19 \mathrm{H} 03259,20 \mathrm{H} 03297$, and $20 \mathrm{H} 05911$ to S.M. S.M. is also supported by the Mitsubishi Foundation. We thank Robert McKenzie, PhD, from Edanz Group (https://jp.edanz.com/ac), for editing a draft of this manuscript.

\section{References}


1. Okabe, K., Sakaguchi, R., Shi, B. \& Kiyonaka, S. Intracellular thermometry with fluorescent sensors for thermal biology. Pflugers Arch - Eur J Physiol 470, 717-731 (2018).

2. Lin, M. Z. \& Schnitzer, M. J. Genetically encoded indicators of neuronal activity. Nat Neurosci 19, 1142-1153 (2016).

3. Miyanari, Y., Ziegler-Birling, C. \& Torres-Padilla, M.-E. Live visualization of chromatin dynamics with fluorescent TALEs. Nat Struct Mol Biol 20, 1321-1324 (2013).

4. Hama, H. et al. ScaleS: an optical clearing palette for biological imaging. Nat Neurosci 18, 15181529 (2015).

5. Susaki, E. A. et al. Whole-Brain Imaging with Single-Cell Resolution Using Chemical Cocktails and Computational Analysis. Cell 157, 726-739 (2014).

6. Yang, B. et al. Single-Cell Phenotyping within Transparent Intact Tissue through Whole-Body Clearing. Cell 158, 945-958 (2014).

7. Ke, M.-T. et al. Super-Resolution Mapping of Neuronal Circuitry With an Index-Optimized Clearing Agent. Cell Reports 14, 2718-2732 (2016).

8. Tainaka, K. et al. Chemical Landscape for Tissue Clearing Based on Hydrophilic Reagents. Cell Reports 24, 2196-2210.e9 (2018).

9. Warner, C. A. et al. An Optical Clearing Technique for Plant Tissues Allowing Deep Imaging and Compatible with Fluorescence Microscopy. Plant Physiol. 166, 1684-1687 (2014).

10. Kurihara, D., Mizuta, Y., Sato, Y. \& Higashiyama, T. ClearSee: a rapid optical clearing reagent for whole-plant fluorescence imaging. Development 142, 4168-4179 (2015).

11. Kurihara, D., Mizuta, Y., Nagahara, S. \& Higashiyama, T. ClearSeeAlpha: Advanced Optical Clearing for Whole-Plant Imaging. Plant and Cell Physiology pcab033 (2021) doi:10.1093/pcp/pcab033.

12. Palmer, W. M. et al. PEA-CLARITY: 3D molecular imaging of whole plant organs. Sci Rep 5, 13492 (2015).

13. Hasegawa, J. et al. Three-Dimensional Imaging of Plant Organs Using a Simple and Rapid Transparency Technique. Plant Cell Physiol 57, 462-472 (2016).

14. Katagiri, Y. et al. The coordination of ploidy and cell size differs between cell layers in leaves. Development 143, 1120-1125 (2016).

15. Sakamoto, Y. \& Matsunaga, S. Deep Imaging of Plant Roots by a Rapid Transparency Technique TOMEI. CYTOLOGIA 82, 221-222 (2017).

16. Nurani, A. M. et al. Deep Imaging Analysis in VISUAL Reveals the Role of YABBY Genes in Vascular Stem Cell Fate Determination. Plant and Cell Physiology 61, 255-264 (2020).

17. Hirai, R. et al. The Progression of Xylem Vessel Cell Differentiation is Dependent on the Activity Level of VND7 in Arabidopsis thaliana. Plants 9, 39 (2019).

18. Musielak, T. J., Slane, D., Liebig, C. \& Bayer, M. A Versatile Optical Clearing Protocol for Deep Tissue Imaging of Fluorescent Proteins in Arabidopsis thaliana. PLOS ONE 11, e0161107 (2016). 
19. Moldovan, G.-L., Pfander, B. \& Jentsch, S. PCNA, the Maestro of the Replication Fork. Cell 129, 665679 (2007).

20. Yokoyama, R., Hirakawa, T., Hayashi, S., Sakamoto, T. \& Matsunaga, S. Dynamics of plant DNA replication based on PCNA visualization. Sci Rep 6, 29657 (2016).

21. Xiong, H. et al. Chemical reactivation of quenched fluorescent protein molecules enables resinembedded fluorescence microimaging. Nature Communications 5, 3992 (2014).

22. Tamaki, S. et al. FT-like proteins induce transposon silencing in the shoot apex during floral induction in rice. Proc Natl Acad Sci USA 112, E901-E910 (2015).

23. Shimamura, M. Marchantia polymorpha: Taxonomy, Phylogeny and Morphology of a Model System. Plant Cell Physiol 57, 230-256 (2016).

24. Kim, C. M., Han, C. \& Dolan, L. RSL class I genes positively regulate root hair development in Oryza sativa. New Phytol 213, 314-323 (2017).

25. Menand, B. et al. An Ancient Mechanism Controls the Development of Cells with a Rooting Function in Land Plants. Science 316, 1477-1480 (2007).

26. Proust, H. et al. RSL Class I Genes Controlled the Development of Epidermal Structures in the Common Ancestor of Land Plants. Current Biology 26, 93-99 (2016).

27. Jang, G., Yi, K., Pires, N. D., Menand, B. \& Dolan, L. RSL genes are sufficient for rhizoid system development in early diverging land plants. Development 138, 2273-2281 (2011).

28. Wu, F. et al. The ABCs of flower development: mutational analysis of $A P 1 /$ FUL -like genes in rice provides evidence for a homeotic (A)-function in grasses. Plant J 89, 310-324 (2017).

29. Ikeda, K., Sunohara, H. \& Nagato, Y. Developmental Course of Inflorescence and Spikelet in Rice. Breed. Sci. 54, 147-156 (2004).

30. Taoka, K. et al. 14-3-3 proteins act as intracellular receptors for rice Hd3a florigen. Nature 476, 332335 (2011).

31. Higo, A. et al. DNA methylation is reconfigured at the onset of reproduction in rice shoot apical meristem. Nat Commun 11, 4079 (2020).

32. Lucob-Agustin, N. et al. WEG1, which encodes a cell wall hydroxyproline-rich glycoprotein, is essential for parental root elongation controlling lateral root formation in rice. Physiol Plantarum $169,214-227$ (2020).

33. Ishizaki, K. et al. Development of Gateway Binary Vector Series with Four Different Selection Markers for the Liverwort Marchantia polymorpha. PLOS ONE 10, e0138876 (2015).

34. Adachi, S. et al. Programmed induction of endoreduplication by DNA double-strand breaks in Arabidopsis. Proceedings of the National Academy of Sciences 108, 10004-10009 (2011).

35. Paxinos, G., \& Franklin, K.B.J. The Mouse Brain in Stereotaxic Coordinates, Second Edition (Academic Press, 2001).

\section{Figures}


a

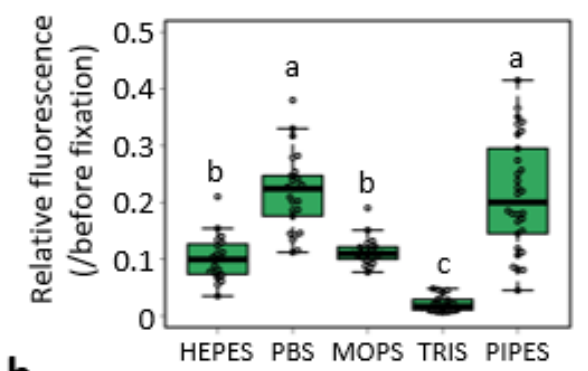

b



d

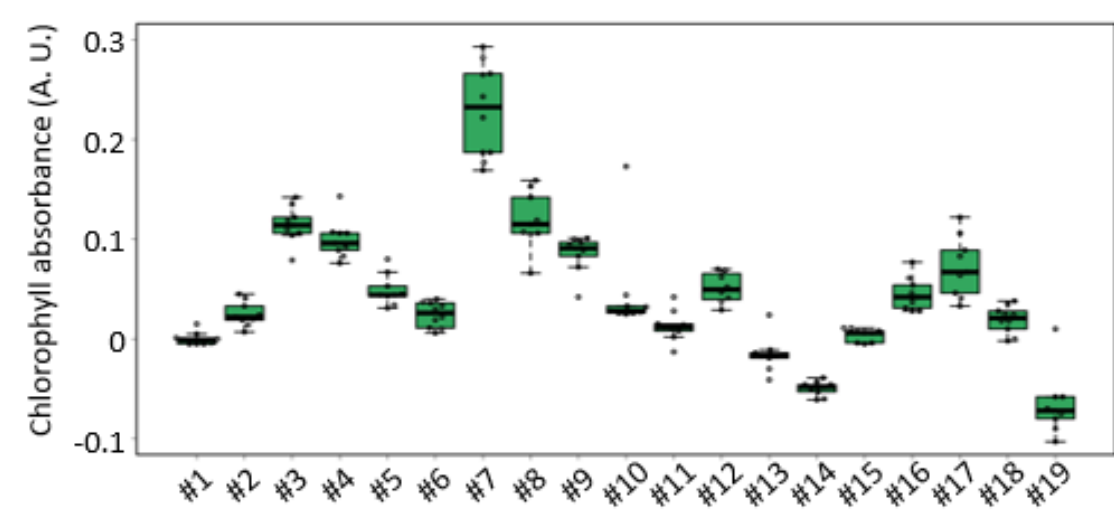

C



$0.1 \%$ FA
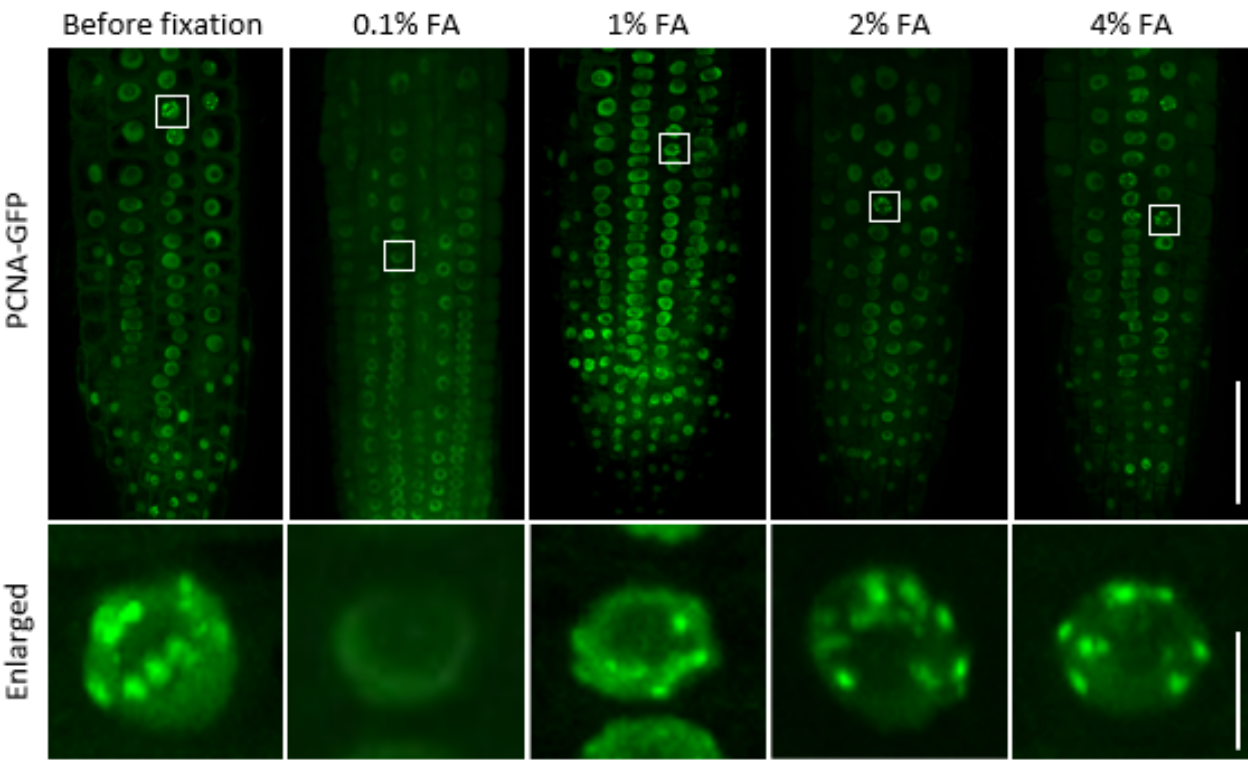

e

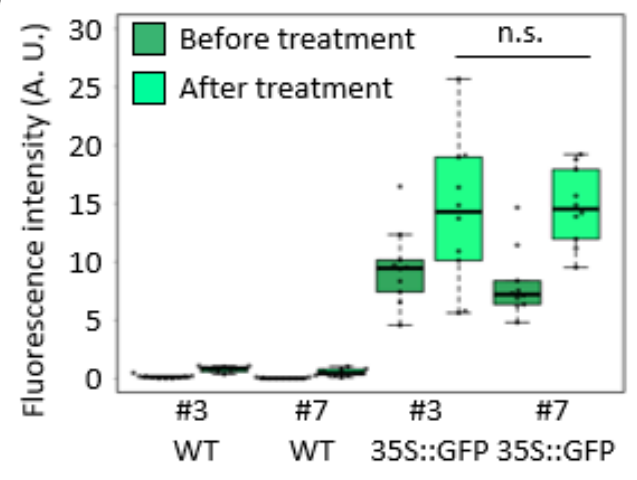

f

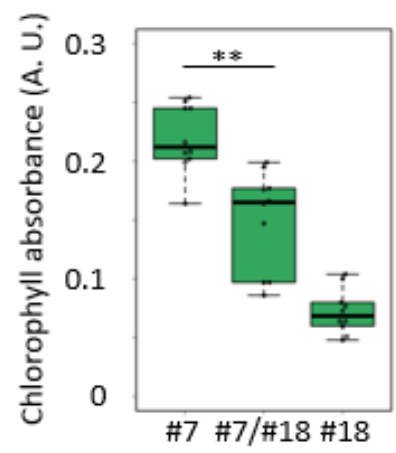

g

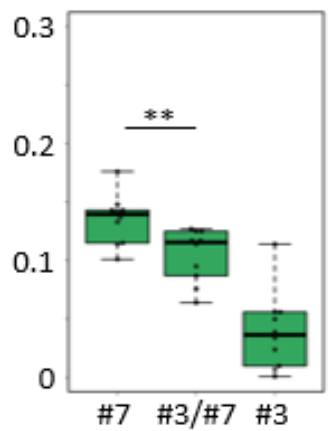

h

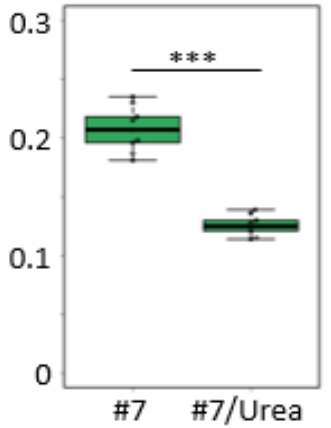

i

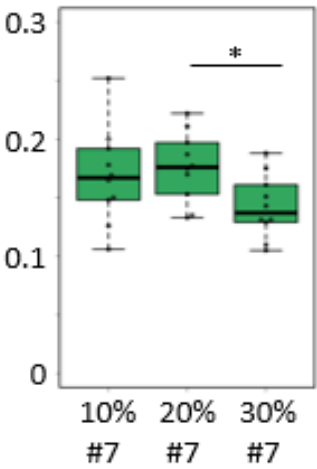

j

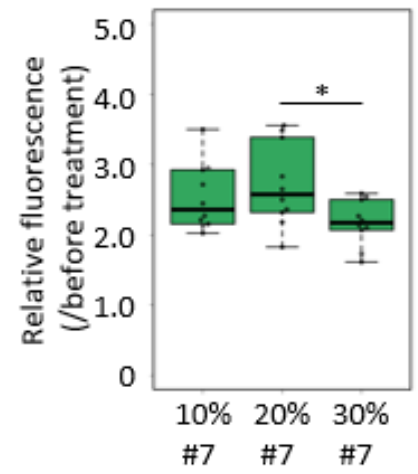

Figure 1

Consideration of fixation and decolorization conditions $\mathrm{a}, \mathrm{b}$ Relative fluorescence intensity was quantified from GFP fluorescence images before and after fixation in various buffers with $2 \%$ FA (a) and in PBS buffer with $1 \%, 2 \%$, or $4 \%$ FA (b). c Confocal micrographs from plants expressing PCNA-GFP fixed in PBS buffer with $0.1 \%, 1 \%, 2 \%$, or $4 \%$ FA captured under the same condition. Lower panels are enlarged images of the area indicated by the white open square in the upper panels. Scale bars $=50 \mu \mathrm{m}$ (top) and $5 \mu \mathrm{m}$ (bottom). $d, f-i$ Chlorophyll absorbance in PBS buffer supplemented with \#1 to \#19 detergents (described 
in Supplementary Table 1) measured using an absorption spectrophotometer. e Fluorescence intensity quantified from fluorescence images of the wild type and plants expressing GFP before and after incubation in PBS buffer supplemented with sodium deoxycholate (\#3) and caprylyl sulfobetaine (\#7). j Relative fluorescence intensity quantified from fluorescence images of GFP expressing plants before and after incubation in PBS buffer supplemented with $10 \%, 20 \%$, or $30 \%$ caprylyl sulfobetaine (\#7).

Significance was determined using the Tukey-Kramer method (a) and unpaired two-sided t-test ( $b, e-j)$. Boxplots show the median (middle bar), 25th and 75th percentiles (upper and lower limits of the box), and $1.5 \times$ interquartile range (whiskers). Each data point is represented by an open circle.

a

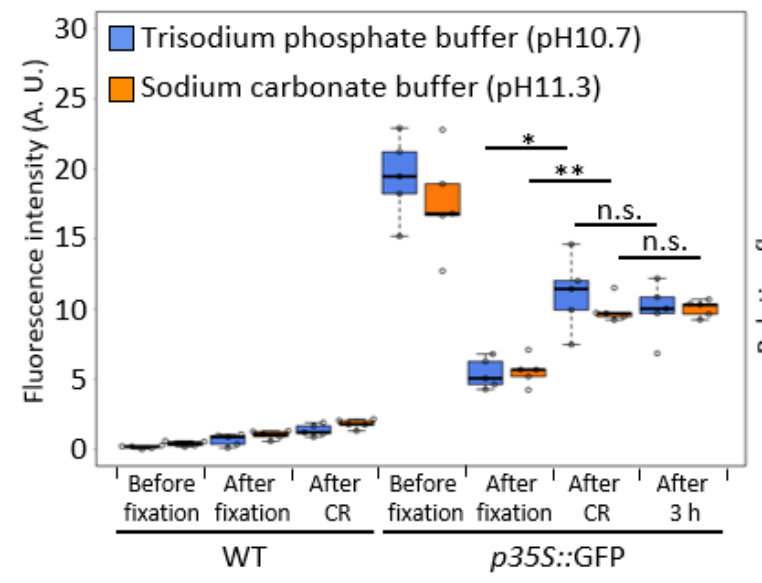

e

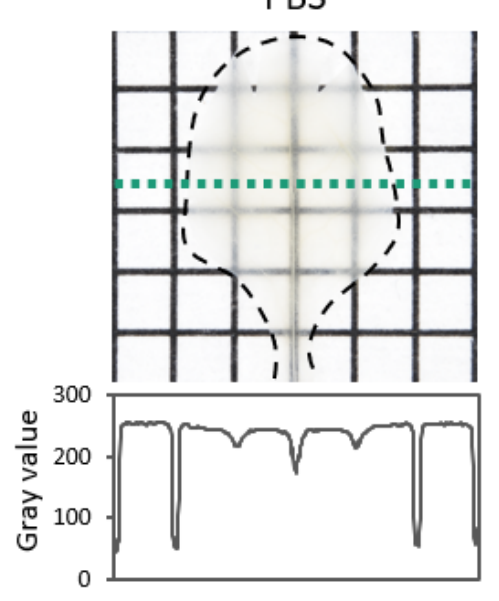

b

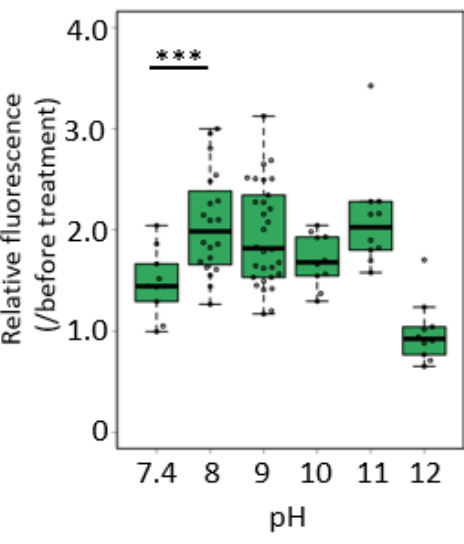

TDE
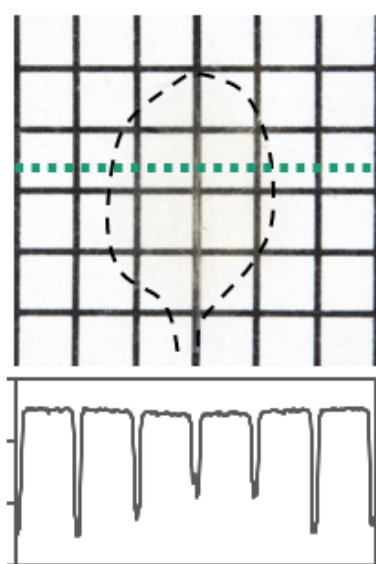

c

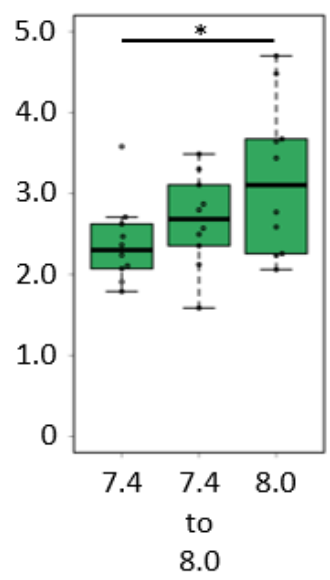

lohexol

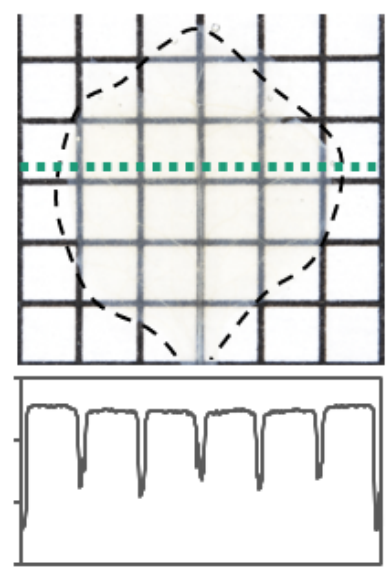

\section{Figure 2}

Assessment of chemical reactivation and mounting condition a Fluorescence intensity quantified from fluorescence images of the wild type and plants expressing GFP before and after fixation and alkaline treatments. $b-d$ Relative fluorescence intensity quantified from fluorescence images of plants expressing GFP before and after incubation in solution of pH 7.4 to 12.0 (b), PBS buffer and sodium phosphate buffer (pH 8.0) (c), and PBS buffer, TDE, and iohexol solution (d). e Decolorized leaves treated with PBS buffer, TDE, and iohexol solution and placed on a grid. Lower panels indicate the intensity profiles along the green dotted line in the upper panels. Boxplots show the median (middle bar), 25th and 75th 
percentiles (upper and lower limits of the box), and $1.5 \times$ interquartile range (whiskers). Each data point is represented by an open circle.

a

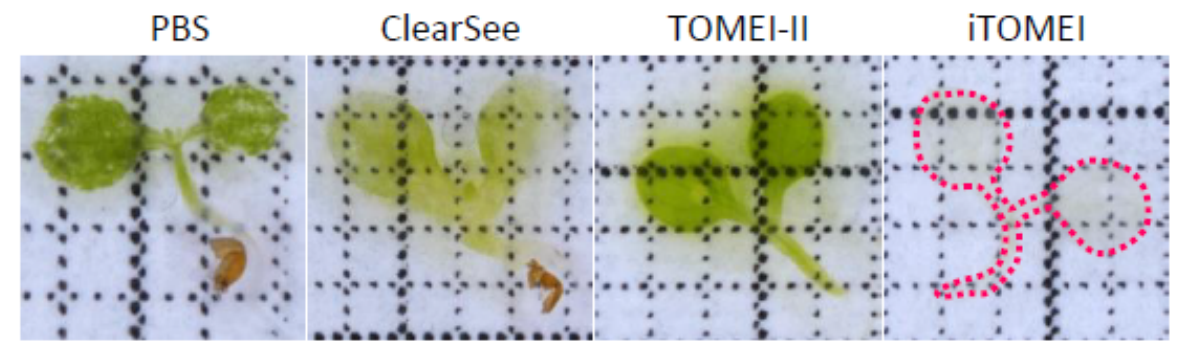

c

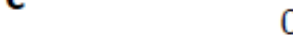

Depth $(\mu \mathrm{m})$

30 b



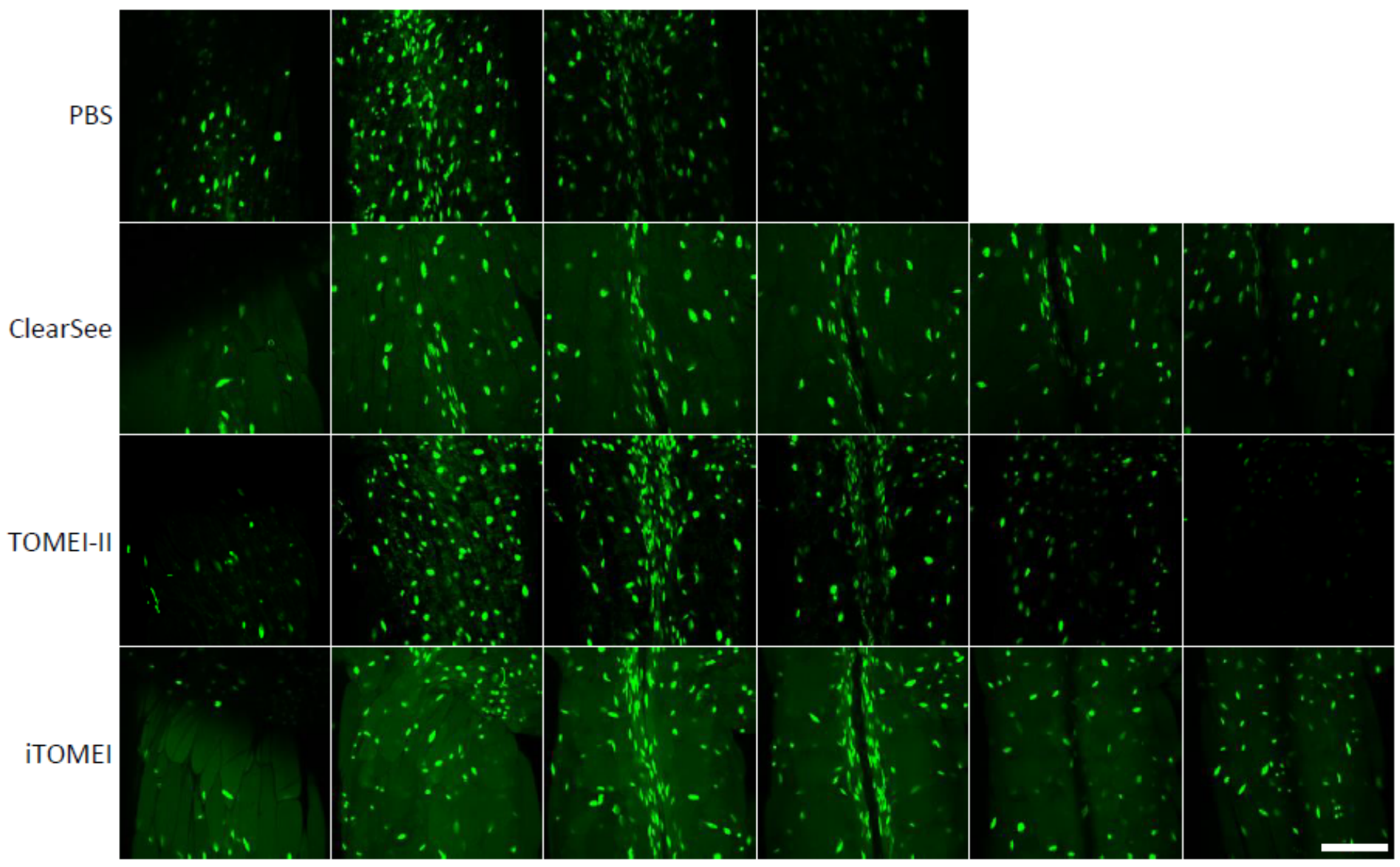

\section{Figure 3}

Comparison of clearing techniques for plant organs a Seedlings treated with four clearing techniques. $b$ Relative fluorescence intensity quantified from fluorescence images of plants expressing GFP before and after various clearing methods. c Leaf of a plant expressing histone H2B-GFP treated with various clearing methods. Confocal optical sections were captured from the dorsal to the ventral surface under the same optical conditions. Scale bars $=100 \mu \mathrm{m}$. Boxplots show the median (middle bar), 25th and 75th 
percentiles (upper and lower limits of the box), and $1.5 \times$ interquartile range (whiskers). Each data point is represented by an open circle, and the mean is represented by a red cross.

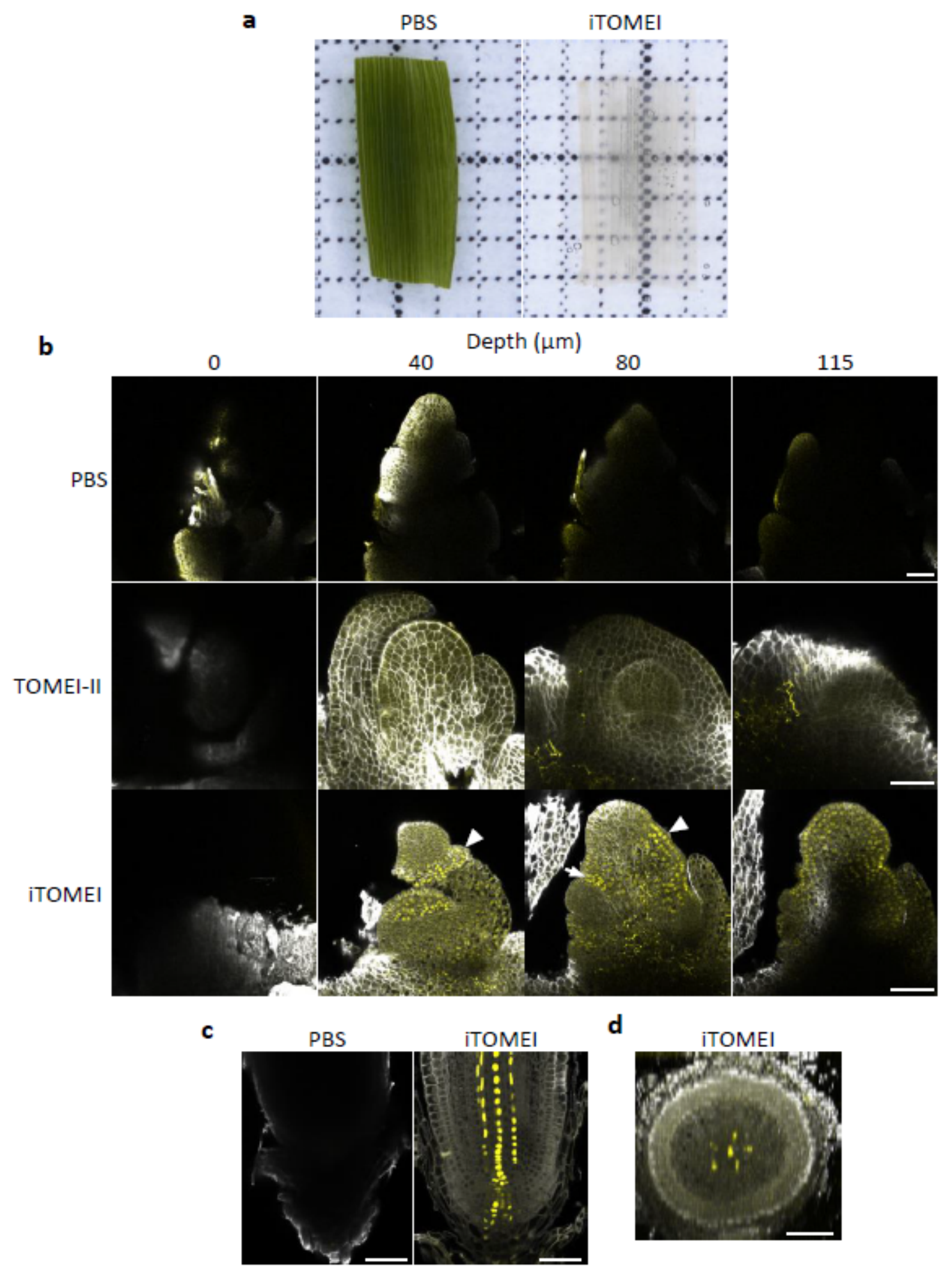

Figure 4

Deep imaging of transparent rice organs using iTOMEl a Leaf of 5-day-old seedling of 0 . sativa treated with PBS and iTOMEI. b Shoot apical meristem of a plant expressing MADS15-mOrange (yellow) stained with SR2200 (white) and treated with PBS, TOMEI-II, and iTOMEI. Confocal optical sections were captured 
under the same optical conditions. The arrow indicates strong mOrange signal in the basal region of a hairy bract. The arrowhead indicates mOrange signal at the incipient primary branch meristem. c, d Root tip of a plant expressing DR5rev::NLS-3xVenus (yellow) stained with SR2200 (white) and treated with PBS and iTOMEI. Confocal longitudinal (c) and transverse optical sections (d) of the root are shown. Scale bars $=50 \mu \mathrm{m}$.

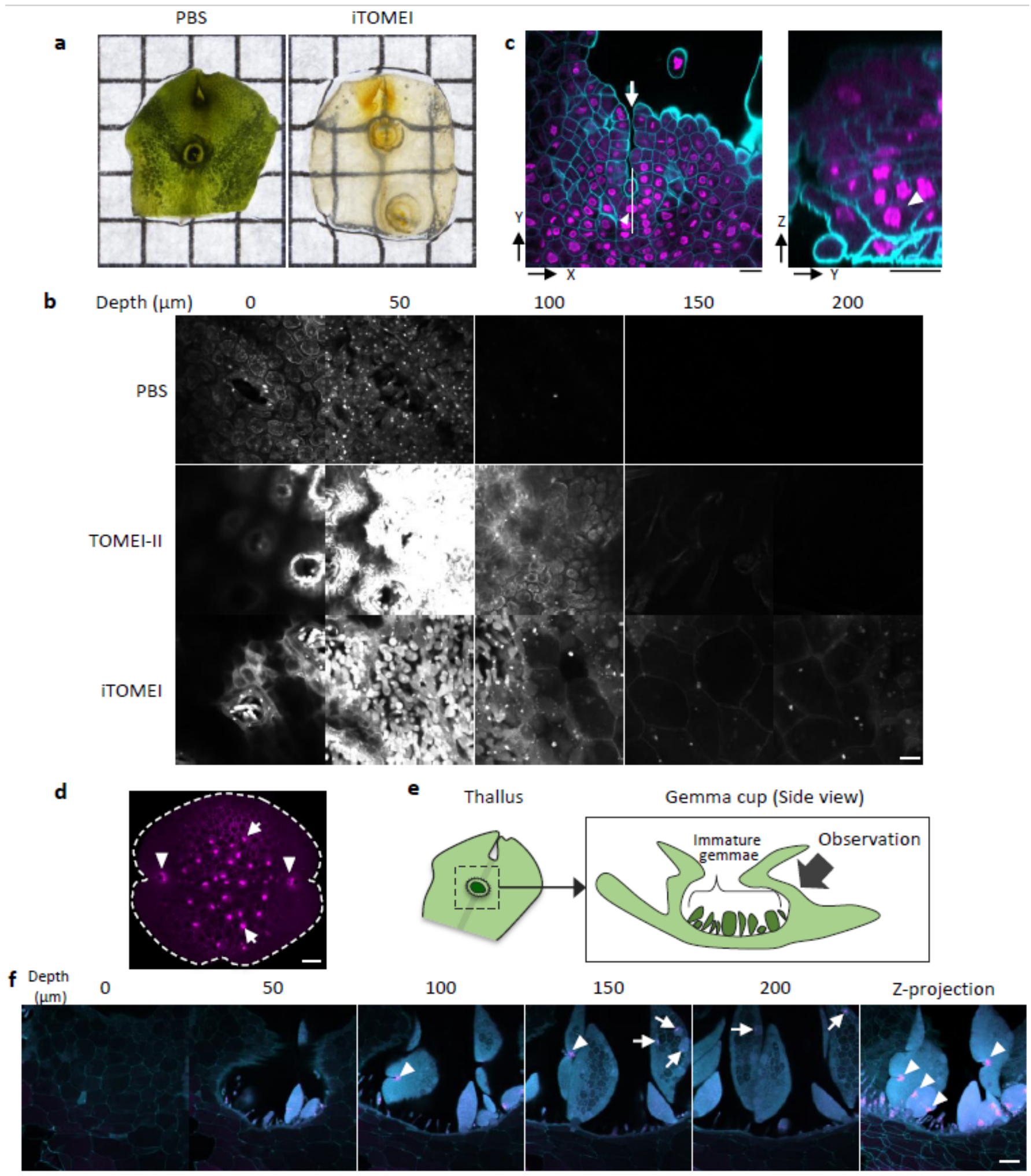

Figure 5 
Deep imaging of transparent organs of M. polymorpha using iTOMEI a Thallus of M. polymorpha treated with PBS buffer and iTOMEI. b Thalli of 2-week-old gemmalings expressing H2B-tdTomato treated with PBS buffer, TOMEI-II, and iTOMEI. Confocal optical sections were captured from the dorsal surface under the same optical conditions. c Thallus of a plant expressing histone H2B-tdTomato (magenta) stained with Calcofluor White (cyan) and treated with iTOMEI. Confocal optical sections were captured from the ventral to the dorsal surface of the apical region and were reconstructed in the $Y-Z$ plane image at a dotted line in $X-Y$ plane image. Arrowheads indicate an apical cell and an arrow indicates an apical notch. d Gemma expressing pMpRSL1::tdTomato-NLS (magenta) treated with iTOMEl. Arrowheads indicate meristematic cells showing high fluorescent signals. The broken line indicates the gemma margin. The arrow indicates a rhizoid precursor cell. d Gemma expressing pMpRSL1:: tdTomato-NLS (magenta) stained with Calcofluor White (cyan) and treated with iTOMEl. e Schematic illustration of a thallus and a gemma cup. When observed with a two-photon excitation microscope, the gemmae were observed through the side wall of the gemma cup. $f$ Gemma cup of a plant expressing pMpRSL1::tdTomato-NLS (magenta) stained with Calcofluor White (cyan) and treated with iTOMEI. The optical sections were captured with a two-photon excitation microscope. The Z-projection image was created from optical sections between the depths of 60 and $120 \mu \mathrm{m}$. Scale bars $=20 \mu \mathrm{m}$ (c) and $50 \mu \mathrm{m}$ $(b, d, f)$.

\section{Supplementary Files}

This is a list of supplementary files associated with this preprint. Click to download.

- 210524SupFigures.pdf 